\title{
To Appreciate the Doppler Sonography as a Simple and Non Invasive Technique for Evaluating Intrarenal Vascular Resistance in Hydronephrosis
}

\author{
Nirmal Kumar Mittal ${ }^{1}$, Renu Goindani ${ }^{2}$, Jagdish Kumar Gupta ${ }^{3}$, Aparna Gupta ${ }^{4}$ \\ ${ }^{1}$ Assistant Professor, ${ }^{2}$ Associate Professor, ${ }^{3}$ Senior Resident, ${ }^{4}$ Senior Resident, Department of Radio-Diagnosis, Amaltas \\ Institute of Medical Sciences, Dewas
}

Corresponding author: Dr. Renu Goindani, Associate Professor, Department of Radio-Diagnosis, Amaltas Institute of Medical Sciences, Dewas

DOI: http://dx.doi.org/10.21276/ijcmsr.2019.4.1.24

How to cite this article: Nirmal Kumar Mittal, Renu Goindani, Jagdish Kumar Gupta, Aparna Gupta. To appreciate the doppler sonography as a simple and non invasive technique for evaluating intrarenal vascular resistance in hydronephrosis. International Journal of Contemporary Medicine Surgery and Radiology. 2019;4(1):A99-A101.

\section{A B S T R A C T}

Introduction: Hydronephrosis simply refers to the dilation of the pelvicalyceal system, which can be of varying severity. The aim of the study was to appreciate the Doppler Sonography as a simple and non invasive technique for evaluating Intrarenal Vascular Resistance in Hydronephrosis.

Material and Methods: This was a hospital based, time bound, and prospective study, done in the Department of Radio diagnosis. The study included all paediatric (<16 yrs) patients, referred to Department of Radiodiagnosis, with strong clinical suspicion of unilateral hydronephrosis and all pre-diagnosed cases of unilateral hydronephrosis by other modalities like Micturating cystourethrography (MCU), CT (whole abdomen and CT- IVU) Renography etc.

Result: The mean post op /follow up RI in hydronephrotic kidney was found to be 0.74 and 0.73 in obstructive and nonobstructive cases respectively. Post op / follow up mean RIR and mean DRI were found to be 1.03 and 0.03 in obstructive cases respectively. Post op/ follow up mean RIR and mean DRI were found to be 1.01 and 0.02 in non obstructive cases respectively.

Conclusion: The colour doppler ultrasonography of intrarenal renal arteries, holds a unique role in imaging workup as well as in optimal management planning, in cases of unilateral paediatric hydronephrosis. The sensitivity, specificity and the diagnostic accuracy is very high, found to be $87.50 \%, 90.00 \%$ and $88.70 \%$ respectively. This is comparable to the other partly invasive /high cost higher modality of imaging.

Keywords: Doppler Sonography, Non-invasive Technique, Intrarenal Vascular Resistance and Hydronephrosis.

Study Designed: Prospective Study

\section{INTRODUCTION}

Hydronephrosis is not equivalent to obstruction of the urinary tract, and only a portion of neonates with hydronephrosis subsequently show true obstruction of urine flow from the kidney due to structural or functional abnormalities. ${ }^{1-2}$ Although urinary tract obstruction is usually manifested by hydronephrosis, but one must remember that several other etiologies may also present as hydronephrosis in children, Vesicoureteral reflux being a common cause. In fact, upon initial screening, it may be indistinguishable from obstructive hydronephrosis.

In diagnostic process it is quite necessary to distinguish between significant obstruction, that requires surgical intervention and nonobstructive cases in which only conservative approach is appropriate in most of the cases. ${ }^{3}$ Hydronephrosis is usually detected on ultrasonography (USG-Gray scale), while neonates or young children with hydronephrosis may occasionally present with abdominal pain or a palpable abdominal mass or complications of obstruction such as urinary tract infection or haematuria.

The study aimed to appreciate the Doppler Sonography as a simple and non invasive technique for evaluating Intrarenal Vascular Resistance in Hydronephrosis.

\section{MATERIAL AND METHODS}

This was a hospital based, time bound, and prospective study, done in the Department of Radio diagnosis of Amaltas Institute of Medical Sciences, Dewas, Madhya Pradesh. The duration of this study was from July -2017 to July -2018 .

\section{Data collection}

a. The study included all paediatric ( $<16$ yrs) patients, referred to Department of Radiodiagnosis, with strong clinical suspicion of unilateral hydronephrosis and all pre-diagnosed cases of unilateral hydronephrosis by other modalities like Micturating cystourethrography (MCU), CT (whole abdomen and CT- IVU) Renography etc. 
b. All symptomatic UTI patients (infants and children) having abdominal pain, painful micturition (dysuria), pyuria, fever or sepsis, were also included.

c. Two groups of patient were made, one with obstructive unilateral hydronephrosis (all pre-diagnosed + our grey scale USG KUB based diagnosed cases) and the other one with non-obstructive unilateral hydronephrosis (all pre-diagnosed + our grey scale USG KUB based diagnosed cases).

d. Determination of RI, RIR, Delta RI were done by renal Doppler ultrasonography, of each patient, in both of the groups then data were collected for comparison.

\section{Inclusion criteria}

The subject of study is age specific. Only neonates, infants and children upto 16 years of age were included in the study. Group 1, Included:-

1. All pre-diagnosed infants and children, with unilateral obstructive hydronephrosis.

2. All infants and children referred to the Department of Radiodiagnosis, AIMS, Dewas with strong clinical suspicion of unilateral obstructive hydronephrosis.

3. All patients, required hospital admission for urinary symptoms [mainly abdominal pain, fever, burning/ painful micturition, and sepsis] or having newly diagnosed unilateral pyonephrosis.

Group 2, Included:-

1. All pre-diagnosed infants and children, with unilateral Non-obstructive hydronephrosis.

2. All infants and children referred to the Department of Radiodiagnosis, AIMS, Dewas with strong clinical suspicion of unilateral non- obstructive hydronephrosis.

3. All patients, required hospital admission for urinary symptoms [mainly abdominal pain, fever, burning/ painful micturition, and sepsis] or having newly diagnosed unilateral pyonephrosis

\section{Exclusion criteria}

1. Patients above 16 years of age.

2. Infants and neonates having bilateral hydronephrosis/ pyonephrosis.

3. Patients having Traumatic or Iatrogenic hydronephrosis.

4. Patients having renal mass lesions.

\section{RESULTS}

The mean post op/follow up RI in hydronephrotic kidney was found to be 0.74 and 0.73 in obstructive and nonobstructive casesrespectively. Post op / follow up mean RIR and mean DRI were found to be 1.03 and 0.03 in obstructive cases

\begin{tabular}{|l|l|c|c|}
\hline S. No & Post op / Follow- up & Obstructive & $\begin{array}{c}\text { Non } \\
\text { Obstructive }\end{array}$ \\
\hline & Mean RI, RIR, Delta RI & & \\
\hline 1 & Mean RI & & \\
\hline & Healthy & 0.71 & 0.72 \\
\hline & Hydronephrotic & 0.74 & 0.73 \\
\hline 2. & Mean RIR & 1.03 & 1.01 \\
\hline 3. & Mean DRI & 0.03 & 0.02 \\
\hline \multicolumn{2}{|c|}{ Table-1: Post operative/follow-up colour Doppler findings } \\
\hline
\end{tabular}

\begin{tabular}{|l|l|c|c|}
\hline S. No & RIR value & No of Pts. & Percentage (\%) \\
\hline 1. & Decreased or Stable & 59 & $95 \%$ \\
\hline 2. & Increased & 3 & $5 \%$ \\
\hline & Total & 62 & $100 \%$ \\
\hline
\end{tabular}

Table-2: Overall result of follow up dopplerscan inall cases (obstructive andnonobstructive both)

respectively. Post op/ follow up mean RIR and mean DRI were found to be 1.01 and 0.02 in non obstructive cases respectively (table-1).

In 59 (95\%) cases out of 62, showed decreased or stable post op /follow-up RIR value as compared to the pre op values. In $3(5 \%)$ cases out of 62 , showed increased post op /follow up RIR value as compared to the pre op values (table-2).

\section{DISCUSSION}

With this background we attempted, to assess the role of Renal Doppler Ultrasound in diagnosing and differentiating between obstructive and nonobstructive unilateral hydronephrosis in infants and children. The primary aim of this study was, to evaluate the role of the Intrarenal Renal artery resistive indices (RI, RIR, DRI) in diagnosing obstruction, as well as discriminating between equivocal obstructive and non obstructive unilateral hydronephrosis.

Prolonged renal obstruction induces hormonal alterations and thereby causes diffuse vasoconstriction of the vascular bed, thus on evaluating different patterns of blood flow in intrarenal arteries (on color doppler) is helpful in differentiatng obstructive from non obstructive pyelocaliectasis. ${ }^{8}$ Unrelieved urinary tract obstruction has been shown to interfere with normal development of the kidney in young children and almost always leads to permanent renal atrophy, termed obstructive uropathy. ${ }^{9}$ Therefore, it is quite necessary to distinguish between significant obstruction, that requires surgical intervention and nonobstructive cases in which only conservative approach is appropriate in most of the cases. ${ }^{10}$ The availability of doppler sonography is apparently a simple, non invasive and well reproducible adjunct to the other partly invasive /high cost diagnostic procedures, commonly used in the radiological assessment of the renal obstruction ${ }^{11}$

\section{CONCLUSION}

The colour doppler ultrasonography of intrarenal renal arteries, holds a unique role in imaging workup as well as in optimal management planning, in cases of unilateral paediatric hydronephrosis. The sensitivity, specificity and the diagnostic accuracy is very high, found to be $87.50 \%, 90.00$ $\%$ and $88.70 \%$ respectively. This is comparable to the other partly invasive /high cost higher modality of imaging.

\section{REFERENCES}

1. Thomas DFM. Fetal uropathy. Brit J Urol 1990; 66(3): 225.

2. Fine RN. Diagnosis and treatment of fetal urinary tract abnor-malities. J Pediatr 1992; 121(1): 333.

3. Elder JS. Antenatal hydronephrosis: fetal and neonatal management. Pediatr Clin North Am 1997; 441299

4. Rao, B.K. and Bryan, P.J. Sonography of acute and chronic renal failure. In Resnick, M.I. and Riftkin, M.D. 
(Eds.) Ultrasonography of the urinary tract, Williams and Wilkins, 1991, 223.

5. Amis, E.S. and Newhouse, J.H. Essentials of uroradiology, Little, Brown and Company, 1991, 233234.

6. Robbins, S.L., Contran, R.S. and Kumar, V. Pathologic basis ofdisease, W.B. Saunders Company, 1989, 10711073.

7. Klahr, S., et al. Urinary tract obstruction. In Brenner, B.M. and Rector, F. (eds.): The Kidney. 3rd ed. Philadelphia, W.B. Saunders Company, 1986, $1443-$ 1490.

8. Onur MR, Cubuk M, Andic C, Kartal M, Arslan G. Role of resistive index in renal colic. Urol Res. 2007; 35(4): 307-12.

9. Tripp BM, Homsy YL. Neonatal hydronephrosis - the contro-versy and management. Pediatr Nephrol 1995; 9(6): 503.

10. Riccabona M. Assessment and management of newborn hydronephrosis. World J Urol 2004;22(2):73-78.

11. Sauvain JL, Pierrat V, Chambers R, Bui Xuan P, Palascak $\mathrm{P}$, Boursheid D, et al. Echography and pulsed Doppler of the arteries of the renal parenchyma in obstructive syndromes and dilatation of the excretory cavities of the kidney. J Radiol 1989; 70(1): 389-98.

Source of Support: Nil; Conflict of Interest: None

Submitted: 01-12-2018; Accepted: 24-12-2018; Published online: 13-03-2019 